\title{
RF Design of the LCLS Gun
}

LCLS-TN-05-3

C.Limborg, Z.Li, L.Xiao, J.F. Schmerge, D.Dowell, S.Gierman, E.Bong, S.Gilevich

February 9, 2005

\section{Summary}

Final dimensions for the LCLS RF gun are described. This gun, referred to as the LCLS gun, is a modified version of the UCLA/BNL/SLAC 1.6 cell S-Band RF gun [1], referred to as the prototype gun. The changes include a larger mode separation (15 MHz for the LCLS gun vs. $3.5 \mathrm{MHz}$ for the prototype gun), a larger radius at the iris between the 2 cells, a reduced surface field on the curvature of the iris between the two cells, $Z$ power coupling, increased cooling channels for operation at $120 \mathrm{~Hz}$, dual $\mathrm{rf}$ feed, deformation tuning of the full cell, and field probes in both cells. Temporal shaping of the klystron pulse, to reduce the average power dissipated in the gun, has also been adopted. By increasing the mode separation, the amplitude of the 0 -mode electric field on the cathode decreases from $10 \%$ of the peak on axis field for the prototype gun to less than $3 \%$ for the LCLS gun for the steady state fields. Beam performance is improved as shown by the PARMELA simulations. The gun should be designed to accept a future load lock system. Modifications follow the recommendations of our RF review committee [2].

Files and reference documents are compiled in Section IV.

\section{I - Beam Dynamics}

A - Mode separation

The RF gun is a two cell device and therefore has two resonant modes. These modes are characterized by their 0and $\pi$ phase shifts between the two cells. The $\pi$ mode (accelerating mode) is resonant at $2856 \mathrm{MHz}$ and the 0mode is resonant at $2852.5 \mathrm{MHz}$ (3.5-MHz below the $\pi$ mode) in the prototype gun. Each mode is approximately $500 \mathrm{kHz}$ wide, corresponding to a loaded Q of about 4000 . When the gun is excited at $2856 \mathrm{MHz}$, the field is dominated by the $\pi$ mode however the 0 -mode is also excited due to finite resonant width of the 0 -mode and finite bandwidth of the driving pulse. The amplitude of the 0mode in the half cell is about $10 \%$ of the $\pi$ mode amplitude in the prototype gun. By increasing the mode separation to $15 \mathrm{MHz}$, this value is reduced to less than $3 \%$. Simulations have shown that the additional voltage from the 0 -mode field does not help reducing the RF emittance. This was a priori unclear. Simulations have shown that, with the larger mode separation, the gun produces a better beam emittance than the prototype design. The 15-MHz mode separation is achieved by modifying the disk of the prototype Gun to a thinner disk with a larger iris opening as described below (Section II-C).

Once steady state is reached, when driven with a long klystron pulse, the 0 -mode phase is close to 90 degrees ahead of that of the $\pi$ mode in the half cell. When driven with the short and shaped pulse, the 0 -mode is still in transition when the $\pi$ mode reaches the designed voltage. The phase difference between the 0 and $\pi$ modes can vary between 40 and 130 degrees as described in paragraph I-C. The variation of emittance with the phase difference is much larger for a $3.5 \mathrm{MHz}$ mode separation than for the $15 \mathrm{MHz}$. This reduced sensitivity to timing adjustements will help tuning the injector beamline. 
The increased mode separation also makes the system less sensitive to changes in the field balance with the gun's body temperature. This should enable the gun to operate stability at both low and high average power.

The frequency shift with cathode plate position is reduced in the LCLS gun. According to Superfish simulations, the LCLS gun is expected to exhibit a $5.2 \mathrm{MHz} / \mathrm{mm}$ frequency shift as the cathode position is varied longitudinally while the prototype gun exhibits a $6.3 \mathrm{MHz} / \mathrm{mm}$ frequency shift.

B- Emittance computations for nominal tuning Simulations were done using PARMELA. The standard $1 \mathrm{nC}$, 10ps long, a1.2mm radius laser pulse and a $0.72 \mathrm{~mm}$.mrad cathode emittance was used. Emittance was optimized principally scanning solenoid and injection phase, i.e. laser timing w.r.t the phase of the accelerating field ( $\pi$-mode). Different maps of field were studied with mode separation ranging from $3.5 \mathrm{MHz}$ to $20 \mathrm{MHz}$. Figure 1 compares simulation results of the prototype gun $(3.5 \mathrm{MHz})$ and the LCLS gun $(15 \mathrm{MHz})$. Shown is the total projected emittance as a function of the phase difference between the 0 -mode and the $\pi$ mode in the $1 / 2$ cell. The optimal "baseline" emittance was computed assuming a 0 voltage field in the 0 -mode. A $0.94 \mathrm{~mm} . \mathrm{mrad}$ emittance was obtained for the $15 \mathrm{MHz}$ and $0.99 \mathrm{~mm}$.mrad for the 3.5 $\mathrm{MHz}$. For a 90 degrees relative phase difference and the nominal field voltages of $12 \mathrm{MV} / \mathrm{m}$ and $3 \mathrm{MV} / \mathrm{m}$ (see I-C), the best emittances obtained were respectively of $0.975 \mathrm{~mm}$.mrad for the prototype gun and $0.945 \mathrm{~mm} . \mathrm{mrad}$ for the LCLS gun. Moreover, the emittance degradation with variation of the relative phase difference is much larger for the $3.5 \mathrm{MHz}$ mode separation case. The tuning and stability tolerances for of the LCLS gun are thus relaxed by adopting a $15 \mathrm{MHz}$ mode separation.

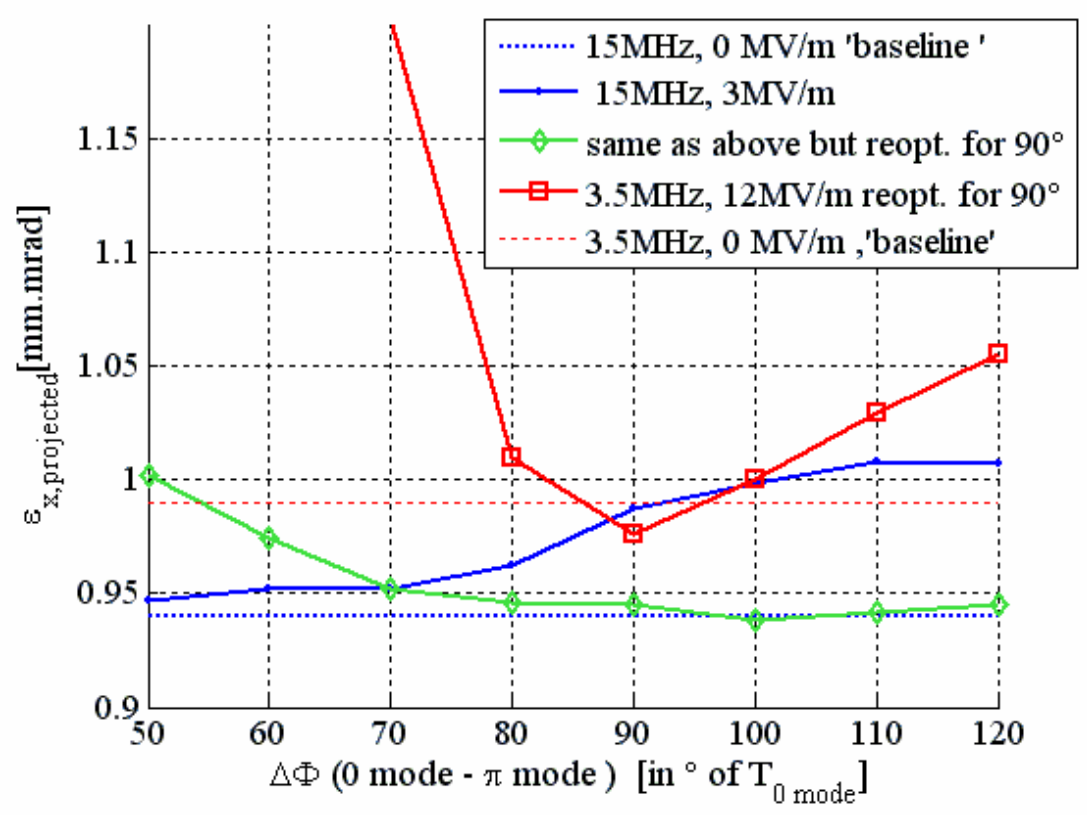

Figure 1- Projected emittance vs phase difference between the $\pi$ and 0 -modes. The baseline levels correspond to the optimum emittance assuming no 0-mode field. 
$\mathrm{C}$ - Amplitude and Phase of the 0-mode field during the klystron pulse Both the $\pi$ and 0modes will be excited by the RF input due to finite resonance width of the modes and finite bandwidth of the klystron pulse. The $\pi$ - and 0 -modes couple to the external drive differently with $\beta=2$ for the $\pi$-mode and $\beta=0.88$ for the 0 -mode. The excitation of the modes in the cavity is simulated using the SLED cavity model [3]. Figure 2 shows the time domain excitation of the $\pi$ - and 0 -modes with two different RF pulses: $3-\mu$ s square pulse and $0.82-\mu$ s shaped pulse.
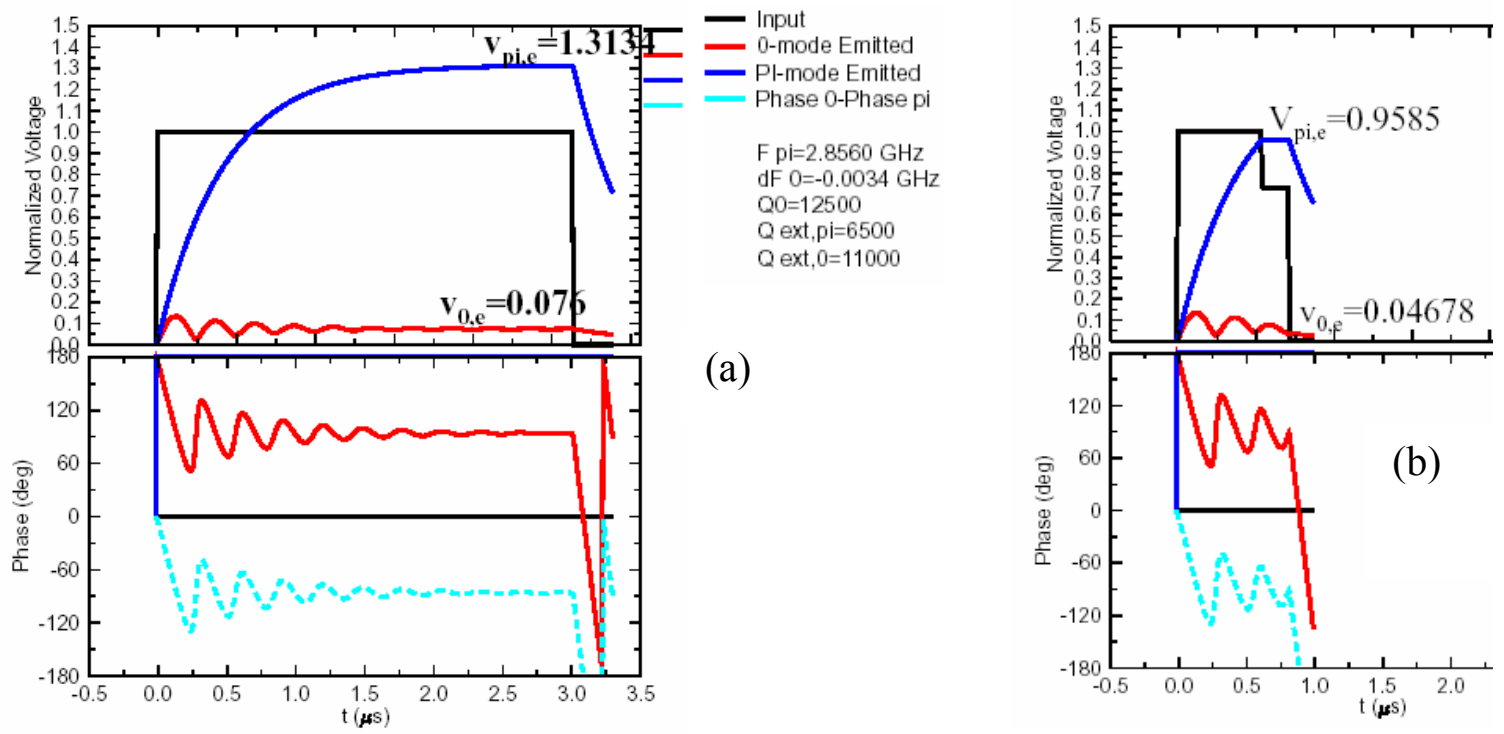

Figure 2: Phase and Amplitude of the $\pi$ and 0modes developing with time for two different klystron pulse shapes ; (a) for a square $3 \mathrm{~ms}$ long pulse ; (b) for a $0.8 \mu$ s klystron pulse with power stepping down from $30 \mathrm{MW}$ to $13 \mathrm{MW}$ after $0.6 \mu$ s was assumed

Using the emitted field information and the RF parameters of the modes obtained using the field solver, the cathode field of the 0mode can be calculated. The ratio of the 0and mode cathode fields for four different designs is shown in the Table 1.

\begin{tabular}{|l|l|}
\hline 0 -mode separation $(\mathrm{MHz})$ & 0 -mode cathode field \\
\hline 3.4 & $10 \%$ \\
\hline 8.0 & $5 \%$ \\
\hline 15.0 & $3 \%$ \\
\hline 20.0 & $2 \%$ \\
\hline
\end{tabular}

Table 1 - Cathode field for the 0 -mode for different mode separation studied

PARMELA simulations show significant improvements in beam emittance with the design of larger mode separation. Therefore, for this and other reasons described in this document, a 15-MHz mode separation is chosen for the LCLS Gun. 


\section{II - Geometry}

Here we describe the modifications incorporated into the LCLS gun which improve its RF properties.

A - Dual Feed

The prototype gun has a single rf feed in the full cell of the gun. An identically sized and shaped hole is machined 180 degrees opposite the coupler hole to reduce the dipole field in the prototype gun. However the presence of the coupler hole and the symmetrizing port induces quadrupole and higher order fields in the coupler cell due to a four-fold symmetry in geometry. In addition, the single rf feed can only compensate for the amplitude term, and the full cell field will still have phase induced multipoles due to the gradient in phase introduced by rf power flowing from only one side of the cell, which are not compensated in the prototype design.

A dual feed can eliminate the dipole field but the quadrupole component remains at a similar level assuming identical coupling holes as used in the single feed gun. In practice, the extra coupling from the second feed leads to a reduced size of each coupling hole which significantly reduces the field perturbation and thus the quadrupole field component. In addition, racetrack cell geometry is used in the new design to minimize the quadrupole field.

Thus a dual feed with proper racetrack geometry eliminates both the dipole and quadrupole fields. The higher order modes are small and therefore negligible.

\section{B- Racetrack shape}

The coupling apertures are designed with coupling coefficient $\beta$ of 2 (see Section II-D), the quadrupole moment $\gamma \beta_{\perp}$ introduced by the couplers is about $4.4 \mathrm{e}-3 / \mathrm{mm}$. In order to correct the quadrupole components, a racetrack cavity shape as shown in Figure 4 is designed. The center offsets of the two arcs are adjusted to minimize the quadrupole field. In the present LCLS gun design, this is reduced to less than $8 \mathrm{e}-5 / \mathrm{mm}$.

The skew quadrupole moment introduced by the laser ports in the half cell was calculated to be approximately $3.8 \mathrm{e}-4 / \mathrm{mm}$. The reduced quadrupole fields in both full and half cells in the new design will not cause significant degradation to the emittance.

PARMELA simulations show that without a racetrack shape in the full cell, the beam would acquire a quadrupole head-tail moment. The horizontal and vertical beam sizes would be varying along the bunch, making the beam matching more difficult in the linac. Simulations show that the racetrack shape entirely suppresses the head-tail quadrupole moment for our nominal beam parameters.

C-Modifications of the cell-to-cell iris dimensions .

There are two modifications to the cell-to-cell iris dimensions to increase the mode separation and to improve the surface field. A comparison of the dimensions of the two guns is given in Table 1. 
To achieve a larger mode separation, the radius of the iris between the two cells was increased from $12.49 \mathrm{~mm}$ to $14.85 \mathrm{~mm}$ and the disk thickness is reduced from $22.05 \mathrm{~mm}$ to $19.05 \mathrm{~mm}$. The shunt impedance was maintained to the same value, $49 \mathrm{M} \Omega / \mathrm{m}$, as that of the prototype gun due to a thinner disk. The new disk dimension appears to produce slightly less RF emittance than does the prototype gun.

\begin{tabular}{|l|l|l|}
\hline Dimension $(\mathrm{mm})$ & Prototype & LCLS RF-Gun \\
\hline iris thickness & 22.0472 & 19.050 \\
\hline iris radius & 12.4968 & 14.850 \\
\hline $1 / 2$ minor axis of iris rounding & 9.525 & 9.525 \\
\hline $1 / 2$ major axis of iris rounding & 9.525 & 12.025 \\
\hline
\end{tabular}

Table 2. Comparison of the characteristics dimensions of iris in the two guns.

The profile of the disk iris was modified from circular to elliptical to reduce the surface field. The two graphs of Figure 3 show that the surface electric field is now lower than the cathode field everywhere. This will possibly help reach higher gradient on the cathode. In the 3D model, the radius of the full cell was reduced to $41.43 \mathrm{~mm}$ for maintaining the frequency in the presence of the power coupling ports and probe holes ports. The radius of the $1 / 2$ cell was reduced to $42.14 \mathrm{~mm}$ to accommodate for the laser ports and probe holes ports.

(a)
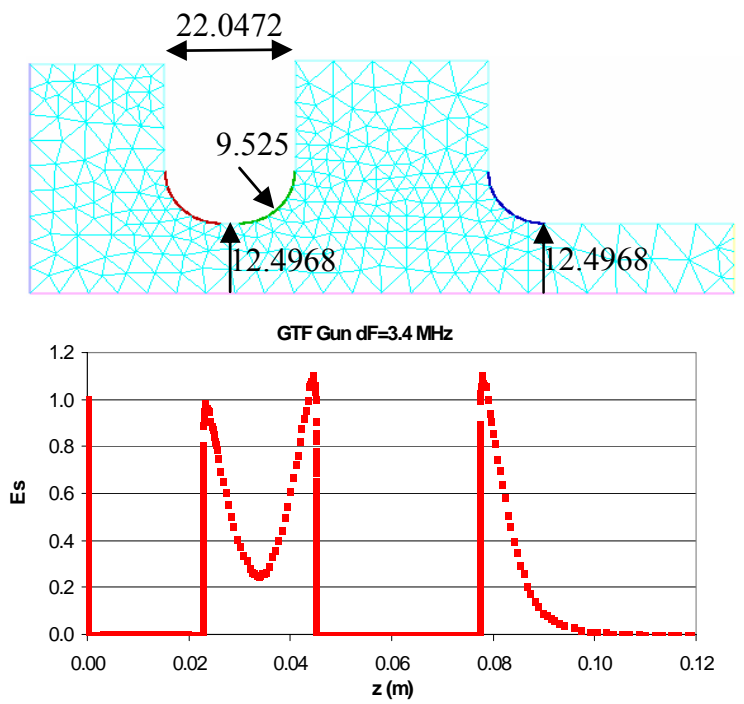

(b)
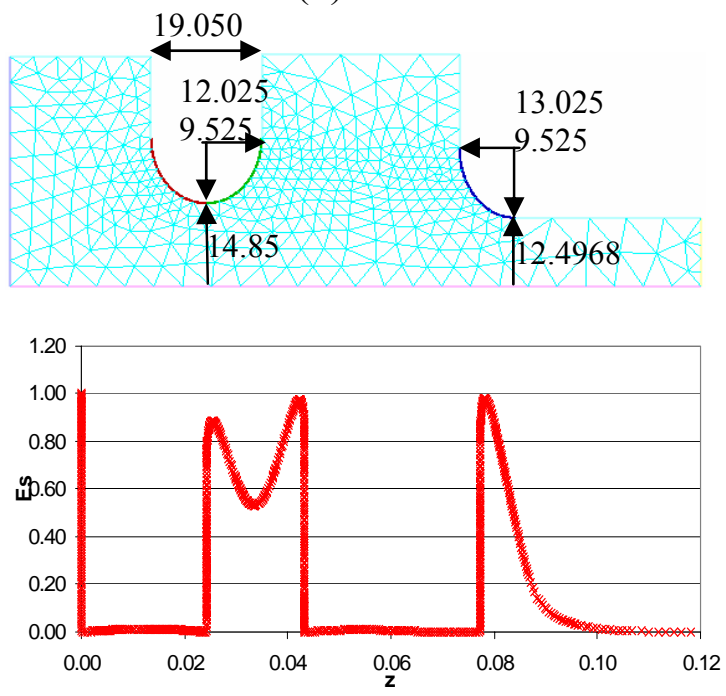

Figure 3- Peak surface field along the gun boundaries for (a) prototype gun and (b) LCLS gun. The field has been normalized to the peak cathode field.

By modifying the disk profile from circular to elliptical, the peak electric surface field is reduced from $11 \%$ higher on the disk to $2 \%$ lower than the field on the cathode. 


\section{D- Z-coupling of RF feeds}

At $120 \mathrm{~Hz}$ operation, the temperature rise at the nose of the power-coupling port in the prototype gun would have been 150 degrees, making it subject to intense thermomechanical fatigue. Based on NLC experience, the temperature rise due to pulsed heating needs to be lowered to less than 50 degrees. In the original $\theta$-coupling design, the cell side of the coupling iris has to be rounded with a radius of $4.7 \mathrm{~mm}$ to reduce the temperature rise to 44 degree. The rounding of the iris along a 3D curve is considered hard to machine. Thus the $\theta$-coupling is replaced by the $z$-coupling in the new design. The rounding on the $\mathrm{z}$-coupling iris is along a straight line and needs a rounding radius of $2.4 \mathrm{~mm}$ to reduce the pulse heating temperature rise to bellow 50 degrees. The $\mathrm{z}$-coupling eases the machining tolerances, simplifies fabrication and reduces pulsed heating.

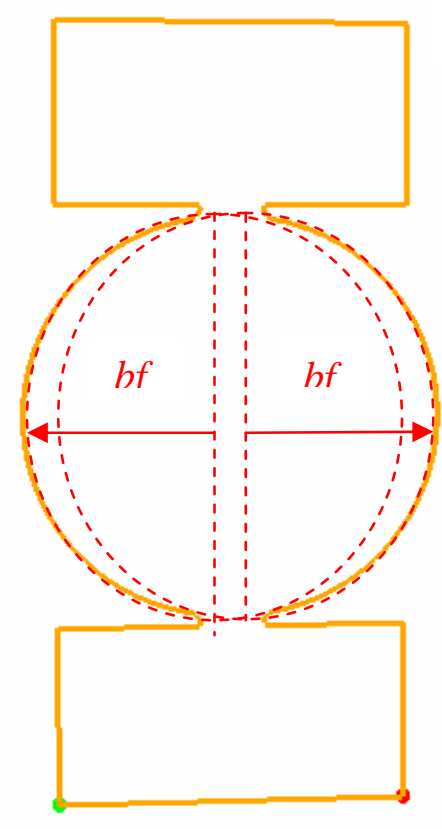

(a)

(b)

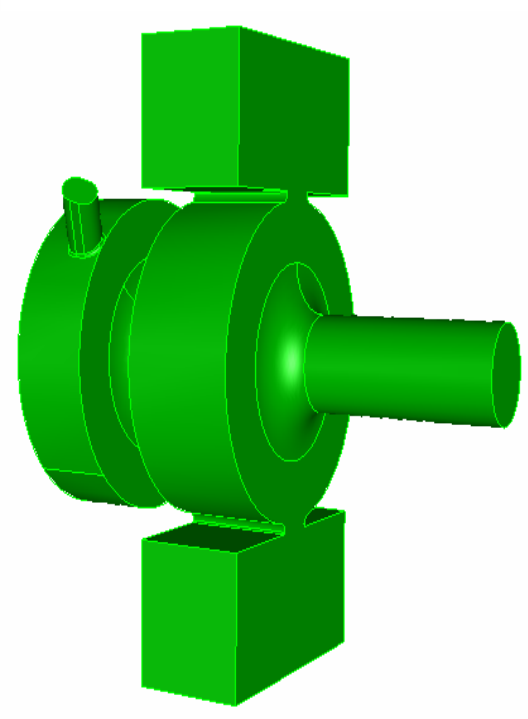

Figure 4-3D model of LCLS gun. (a) racetrack shape in full cell; (b) shows Z-coupling ports on both rf feeds in the interior model of the gun.

E- Adjustment of beta value

By adjusting the gap of the z-coupling slot to $13.2 \mathrm{~mm}$, the beta value was maintained to a value of two. The over-coupling is beneficial to pulsed heating, operating gradient and sensitivity of RF phase to changes of the gun temperature. A more detailed discussion on the choice of a $\beta$ of 2 is given in [5]. 


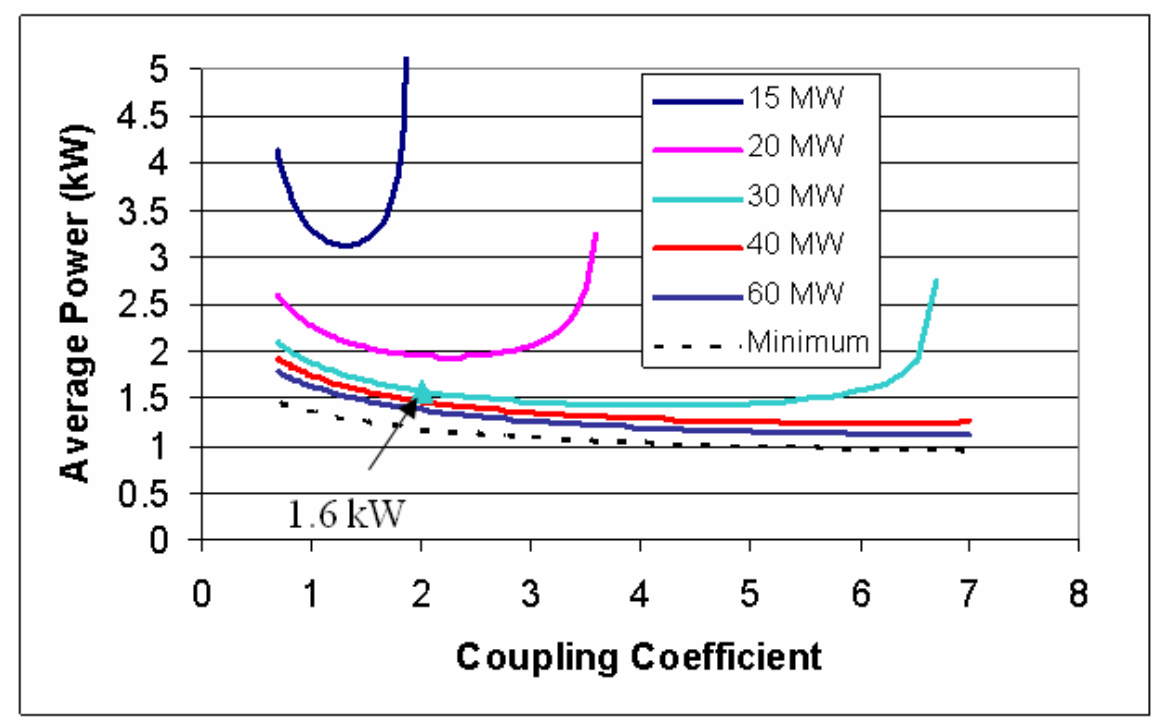

Figure 5 - Graph of average power dissipated in the gun versus the coupling coefficient for different values of peak power incident on the gun from the klystron. The klystron pulse was square and its length was adjusted to meet the peak power level given in the legend.

\section{F- Laser port holes}

The two laser port holes are at $180^{\circ}$ from one another in the $1 / 2$ cell and at 45 degrees from the horizontal plane. The laser beam will go through an elliptical aperture with major axis of $11 \mathrm{~mm}$ and the minor axis of $8 \mathrm{~mm}$.

The optical design of the Injector launch system has the ability to adjust the beam size and position on the photocathode, and it affects the size and position of the beam inside the cathode assembly of the gun. The following table represents the largest numbers of the beam radius at different distances from the cathode. The calculations were done with the assumption that the laser beam bandwidth is $3 \mathrm{~nm}$.

\begin{tabular}{|l|l|l|}
\hline Distance from the cathode & Beam Radius Horizontal & Beam Radius Vertical \\
\hline $10 \mathrm{~mm}$ & $0.91 \mathrm{~mm}$ & $2.94 \mathrm{~mm}$ \\
\hline $20 \mathrm{~mm}$ & $1.3 \mathrm{~mm}$ & $2.88 \mathrm{~mm}$ \\
\hline $55.61 \mathrm{~mm}$ & $2.79 \mathrm{~mm}$ & $2.6 \mathrm{~mm}$ \\
\hline $110 \mathrm{~mm}$ & $5.11 \mathrm{~mm}$ & $2.3 \mathrm{~mm}$ \\
\hline
\end{tabular}

Table 3- Laser beam dimensions going through laser ports; the reference, $\mathrm{z}=0$, is the cathode plane

In order to keep the size of the window (located at the distance $\mathrm{L}_{\mathrm{w}}=55.61 \mathrm{~mm}$ from the cathode) as small as possible the beam steering over the cathode will be achieved by changing the angle and position of the steering mirrors. This allows moving the laser beam on the cathode while keeping it centered on the laser vacuum window. With this 
technique, we avoid having to open the window size. In the vertical plane the beam incident angle is $90^{\circ}$. (Reference [6].)

In order to provide the maximum vertical steering on the cathode (for a maximum beam diameter $\mathrm{S}=3 \mathrm{~mm}$ ) the incident angle should be changed by

$\Delta \alpha_{\mathrm{vertical}}=\arctan \left(\mathrm{S} / \mathrm{L}_{\mathrm{w}}\right)=3.07^{0}$

In the horizontal plane the incident angle is $\theta=22.5^{0}$

In order to provide the maximum horizontal steering on the cathode $S=3 \mathrm{~mm}$ the incident angle should be changed by

$\Delta \alpha_{\text {horizontal }}=\arcsin \left[\sin \theta /\left(\mathrm{L}_{\mathrm{w}} / \mathrm{S}+\cos \theta\right)\right]=1.12^{0}$

PARMELA simulations show that the skew head-tail quadrupole moment does not show on the beam for our nominal parameters. The introduction of the laser port holes in the $1 / 2$ cell slightly changed the tuning but did not degrade either the slice or the projected emittance.

F- Probe holes

Two rf probe ports in each cell for maintaining some symmetry. They will be located at the outer diameter of each cell with small port holes which negligibly perturb the axial field, but in the region of high electrical field for maximum signal. The first pair of probe holes (in the $1 / 2$ cell) will be located at $\mathrm{z}=13 \mathrm{~mm}$ and the second pair (in the full cell) will be at $\mathrm{z}=53.3 \mathrm{~mm}$.

Computations show that with a $5 \mathrm{~mm}$ diameter hole the temperature rise will be of $36^{\circ} \mathrm{C}$ for the probe holes of the $1 / 2$ cell and of $45^{\circ} \mathrm{C}$ for those of the full cell with $0.5 \mathrm{~mm}$ rounding curvature. These numbers are totally acceptable.

The port hole dimensions will be smaller than $5 \mathrm{~mm}$ diameter. It was checked that the quadrupole moment induced by the presence of $5 \mathrm{~mm}$ diameter probe holes is smaller than $7.10^{-5}$ in the $1 / 2$ cell and $8.10^{-5}$ in the full cell. Those are negligible. We believe that the other multipole terms are negligible (in particular the octupole one).

Each probe's coupling should be between -50 to $-60 \mathrm{~dB}$, and should not intrude into the gun body. The probe holes dimensions will be computed to match the $50 \mathrm{Ohms}$ of the coaxial line transporting the signal. Final dimensions will be given in revision 1 of this document. The $1 / 2$ cell radius and the dimensions of the racetrack in the full cell will then have to be adjusted.

The probe holes will be at 90 degrees from the laser ports in the $1 / 2$ cell. In the full cell, the final azimuthal location of the probe holes is still undecided as we also need to accommodate the push-pull studs for deformation tuning. The precise locations can be decided during the engineering phase of the design. Three possible orientations are: 
1- probe holes at 60 degrees from coupler ports, then the pair of push-pull studs at 60 degrees

2- probe holes at 90degrees from coupler ports, then 2 pairs of push-pull studs at 45 degrees (requires deformation with same force on each stud)

3 - pair of push-pull studs at 90 degrees from coupler ports, then pair of probe holes at 45 degrees; this will work only if the probe holes are very small

G- LCLS gun dimensions

\begin{tabular}{|l|l|}
\hline Dimension & Value \\
\hline \hline Race track arc radius bf & $41.4338 \mathrm{~mm}$ \\
\hline \hline Race track arc separation d & $2.8 \mathrm{~mm}$ \\
\hline Race track cell length zf & $34.0106 \mathrm{~mm}$ \\
\hline Half cell radius zh & $42.1486 \mathrm{~mm}$ \\
\hline Half cell length zh & $24.2570 \mathrm{~mm}$ \\
\hline RF coupling hole size (slot width) wc & $13.2 \mathrm{~mm}$ \\
\hline RF coupling hole rounding radius r1 on outside & $0.56 \mathrm{~mm}$ \\
\hline RF coupling hole rounding radius r2 on inside & $2.4 \mathrm{~mm}$ \\
\hline Beampipe radius api & $12.4968 \mathrm{~mm}$ \\
\hline \hline Cell iris radius a & $14.85 \mathrm{~mm}$ \\
\hline Disk thickness t & 19.05 \\
\hline Disk elliptical rounding radius $\mathrm{r}$ & 9.525 \\
\hline Laser port hole size (slot length) 11 & $11.0 \mathrm{~mm}$ \\
\hline Laser port hole radius of curvature rl & $4.0 \mathrm{~mm}$ \\
\hline Laser port hole size (slot width) $2 \mathrm{rl}$ & $8.0 \mathrm{~mm}$ \\
\hline Laser port hole rounding radius $\mathrm{r} 3$ & $0.762 \mathrm{~mm}$ \\
\hline Laser port angle & $22.5 \mathrm{degree}$ \\
\hline \hline Waveguide & $34.0106 * 72.136 \mathrm{~mm}^{2}$ \\
\hline
\end{tabular}

Table 4 - LCLS gun Dimensions

\begin{tabular}{|l|l|}
\hline $\mathrm{f} \pi$ & $2.856 \mathrm{GHz}$ \\
\hline $\mathrm{df}=\mathrm{f} \pi-\mathrm{fo}$ & $15 \mathrm{MHz}$ \\
\hline$\beta$ & 2 \\
\hline Rs & $49 \mathrm{MOhms} / \mathrm{m}$ \\
\hline Qo & 12000 \\
\hline Qloaded & 4000 \\
\hline $\mathrm{E}$ & $>120 \mathrm{MV} / \mathrm{m}$ \\
\hline
\end{tabular}

Table 5 - Characteristics of the LCLS gun 

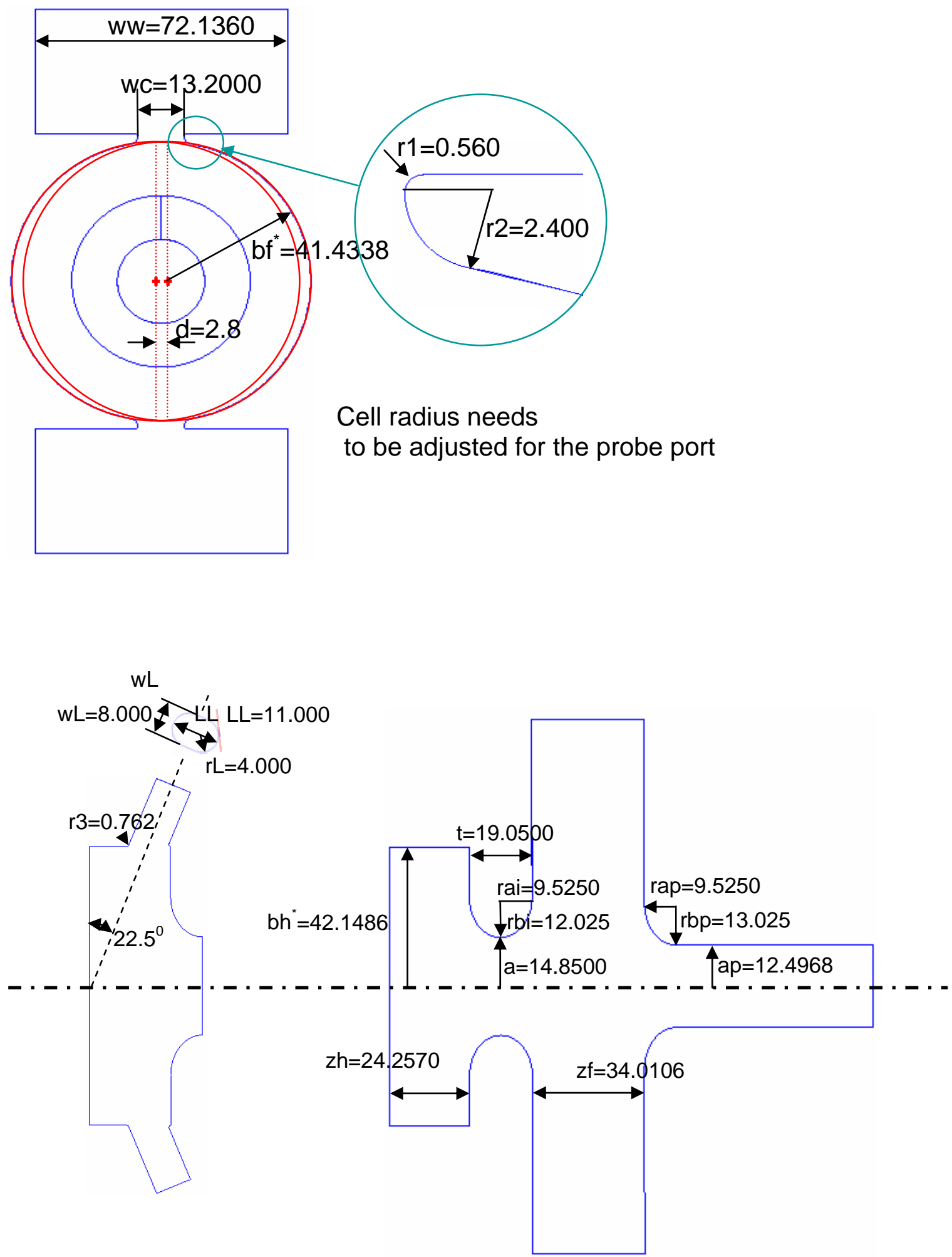

Figure 6 - To scale drawings of the LCLS gun defining the parameters given in Table 4. 


\begin{tabular}{|l|l|l|l|}
\hline $\begin{array}{l}\text { dimension increase } \\
0.010-\mathrm{mm}\end{array}$ & $\begin{array}{l}\mathrm{dF} 0 \\
(\mathrm{kHz})\end{array}$ & $\begin{array}{l}\mathrm{dF} \pi \\
(\mathrm{kHz})\end{array}$ & $\begin{array}{l}\text { Field Balance } \\
(\mathrm{bf}-\mathrm{bh}) / \mathrm{bf}-1)(\%)\end{array}$ \\
\hline $\mathrm{bh}$ & -457.1 & -251.6 & 4.6 \\
\hline $\mathrm{bf}$ & -268.5 & -447.5 & -5.1 \\
\hline $\mathrm{a}$ & 94.0 & 133.7 & -0.3 \\
\hline $\mathrm{t}$ (fixed total length) & 47.9 & 36.0 & -0.2 \\
\hline $\mathrm{zh}$ & -32.7 & -17.4 & 0.2 \\
\hline $\mathrm{zf}$ & -16.0 & -26.1 & -0.4 \\
\hline $\mathrm{d}$ & -80.0 & -134.0 & 1.4 \\
\hline wc & -9.0 & -9.9 & 0.3 \\
\hline
\end{tabular}

Table 6 . The sensitivity of mode frequencies and field balance to gun dimensions. The mechanical tolerances will be determined once the tuning range of the push-pull has been defined in a future revision of this report.

\section{III - Thermal analysis}

A - Cooling channels

Steady state thermal analysis has been performed on the LCLS gun using ANSYS assuming a $4 \mathrm{~kW}$ heat load $[4,5]$. The $4 \mathrm{~kW}$ heat load assumes $120 \mathrm{~Hz}$ operation at 140 $\mathrm{MV} / \mathrm{m}$ peak field on axis with a $3 \mu$ s long klystron pulse and a gun filling time of approximately $600 \mathrm{~ns}$.. The results of the analysis indicate four water cooling channels are required to keep the stress and stress induced distortions under specification. Additional cooling may be required to eliminate deleterious pulsed heating effects. The final location and size of the cooling channels will be established in the detailed engineering design.

A temperature monitor (for example thermocouple) will be required in a feedback loop to keep the gun body temperature constant and the stresses under specification. The Injector RF Technical Review Committee [2] advised controlling the gun resonance frequency by measuring the reflected $\mathrm{rf}$ power and to minimize it by regulating the gun cooling water temperature. This technique has been used on other accelerator systems and should be implemented into the gun control system.

The gun design should include thermocouples at several key locations throughout the gun's volume, in order to monitor and control the thermal gradients and gun dimensions. Sensors capable of measuring critical dimensions are desirable, and would assure the gun balance and field shape remains as specified during high power operation.

B - Reduction of average power by shaping the RF pulse One way to reduce the steady state and pulsed heating effects of the $120 \mathrm{~Hz}$ rf gun is reduce the amount of power dissipated in the gun body. The most direct method to achieve this is to shape the incident rf pulse so that the field no longer builds up exponentially to the final value which requires several time constants [5]. The idea is to reach the steady state field value in the gun faster than the time constant of the gun would allow when using a flat incident rf pulse. By first increasing the incident power by about 
a factor of three, and then, rapidly decreasing it when the field reaches the desired value in the gun, the field build up time can be reduced by more than a factor of three. With this technique the heat load is decreased by more than a factor of three. In addition the rf coupling coefficient can be increased from the typical critically coupled designs to overcoupled which helps reduce the field build up time. Increasing the coupling coefficient from 1 to 2 reduces the heat load by another $25 \%$ and still limits the reflected power and coupling hole size to realistic values. Therefore, to allow this mode of operation, the gun should be built with a coupling coefficient of 2 and the cooling channels designed to dissipate $4 \mathrm{~kW}$ average power.

IV - References

Files of reference

- ANSYS file

- $\quad$ Superfish file (for lcls gun )

http://www-ssrl.slac.stanford.edu/lcls/photoinjector/internals/rfgun/sf7 lcls.txt

- Solid model

http://www-ssrl.slac.stanford.edu/lcls/photoinjector/internals/rfgun/guncavity.par

- PARMELA file (for gun_1: 2 versions 2D and complete 3D)

http://www-ssrl.slac.stanford.edu/lcls/photoinjector/internals/optics/

Stored on web site at :

http://www-ssrl.slac.stanford.edu/lcls/photoinjector/internals/rfgun/

References:

[1] D.Palmer PhD thesis “The next Generation Photoinjector", June 1998

[2 ] Report of the LCLS Injector Technical Review Committee, SLAC Memo, dated November 22, 2004,

http://www-ssrl.slac.stanford.edu/lcls/photoinjector/internals/internals.html\#RF

[3] Z.D.Farkas, "SLED: A Method of doubling SLAC's Energy", SLAC-PUB-1453

[4] R.F. Boyce, D.H. Dowell, J. Hodgson, J.F. Schmerge, N. Yu, "Design

Considerations for the LCLS RF Gun.", LCLS-TN-04-4,

[5] J.Schmerge, "Reducing the Heat Load on the LCLS $120 \mathrm{~Hz}$ RF Gun with RF Pulse Shaping", LCLS-TN-02-7

[6] A. Miahnahri, LCLS Injector Technical Note: LCLS INJ-05-01, "Cathode Gun Assy./Laser Port Clearance Study"

http://www-ssrl.slac.stanford.edu/lcls/photoinjector/internals/laser/notes/inj-05-01.pdf 